\title{
Periodic Domain Boundary Ordering in a Dense Molecular Adlayer: Sub-saturation Carbon Monoxide on Pd(111)
}

\author{
Pan $\mathrm{Xu}$, ${ }^{\text {a }}$ Shizhong Liu, ${ }^{\mathrm{a}}$ Sung-Young Hong, ${ }^{\mathrm{b}}$ Ping Liu, ${ }^{\mathrm{b}}$ \\ Michael G. White ${ }^{\mathrm{a}, \mathrm{b}}$ and Nicholas Camillone III $\mathrm{b}, *$ \\ ${ }^{a}$ Department of Chemistry, Stony Brook University, Stony Brook, NY 11794 \\ ${ }^{\mathrm{b}}$ Chemistry Division, Brookhaven National Laboratory, Upton, NY 11973
}

\begin{abstract}
We describe a previously unreported ordered phase of carbon monoxide adsorbed on the (111) facet of single crystal palladium at near-saturation coverage. The adlayer superstructure is identified from low energy electron diffraction to be $\mathrm{c}(16 \times 2)$ with respect to the underlying $\operatorname{Pd}(111)$ surface net. The ideal coverage is determined to be $0.6875 \mathrm{ML}$, approximately $92 \%$ of the $0.75-M L$ saturation coverage. Density functional theory calculations support a model for the molecular packing characterized by strips of locally-saturated $(2 \times 2)$ regions, with the $\mathrm{CO}$ bound near high-symmetry surface sites, separated by antiphase domain boundaries. The structure exists in a narrow coverage range and is prepared by heating the saturated adlayer to desorb a small fraction of the CO. Comparison of the $\mathrm{c}(16 \times 2)$ domain-boundary structure with structural motifs at lower coverages suggests that between 0.6 and $0.6875 \mathrm{ML}$ the adlayer order may be more strongly influenced by interadsorbate repulsion than by adsorption-site-specific interactions. The system is an example of the structural complexity that results from the compromise between adsorbate-substrate and adsorbate-adsorbate interactions.
\end{abstract}

Keywords: palladium; carbon monoxide; low energy electron diffraction (LEED); temperature programmed desorption (TPD); density functional theory (DFT); antiphase domain boundary

* Corresponding author E-mail: nicholas@bnl.gov 


\section{Introduction}

Carbon monoxide adlayers on metal surfaces have, since the early days of ultrahighvacuum surface science, served as model systems for the study of the molecule-surface and molecule-molecule interactions that determine surface structure, dynamics and chemistry. The detailed investigation of the rich structural diversity of $\mathrm{CO}$ adlayers on low-index palladium surfaces spans nearly five decades [1-11]. By the late 1980's the CO/Pd(111) system in particular had been studied with a variety of methods, and the progression of adlayer structures and molecular binding sites appeared to be fairly well established [12]. Some models for the adsorption geometries at high coverage-between 0.5 and 0.75 -ML (saturation) - featured molecules generally displaced from high symmetry surface sites (e.g., Figure 1e), suggesting a continuous compression of the adlayer with increasing surface density, minimally influenced by adsorption-site-specific adsorbate-substrate interactions [3,12,13].

However, discussion regarding adsorption geometries continued [14]. The continuouscompression model was thought to be inconsistent with the sharp and limited number of absorption bands observed in infrared (IR) spectroscopy, which were suggested instead to indicate population of only a small number of adsorption sites [6]. A model based on sitespecific adsorption and periodic ordering of antiphase domain boundaries, also known as solitons, was proposed to account for both the low-energy electron diffraction (LEED) and IR data [15]. In some of the early high-coverage models, the structures involved packing at

distances of $\sqrt{3} / 2 d_{\mathrm{Pd}}=2.38 \AA$, where $d_{\mathrm{Pd}}$ is the Pd nearest neighbor distance (e.g., Figure 1c). This is significantly smaller than the $\sim 3.4-\AA$ van der Waals diameter of CO [16], and it is fair to question whether such an arrangement is energetically favorable compared to the "compression" model. A subsequent soliton model involving exclusively bridge- and near-bridge-site adsorption 
and heavy antiphase domain boundaries (e.g., Figure 1d)-within which a subset of the molecules are packed more closely together relative to the average density-reduced the extent of tight packing for the 0.5-0.6-ML coverage range [6] ( $c f$. Figures $1 \mathrm{c}$ and 1d), and has gained support from recent molecularresolution scanning tunneling microscopy (STM) of $\mathrm{CO}$ adsorbed on the (111) facets of $\mathrm{Pd}$ nanocrystals [10].

Understanding of the adsorption-sitegeometry tendencies at high coverages remains unsettled, however. For example, at $0.5 \mathrm{ML}$, experiments and calculations have been interpreted to indicate that a single 2-molecule $\mathrm{c}(4 \times 2)$ unit mesh contains COs: (i) adsorbed exclusively at bridge sites [6] (Figure 1a), (ii) one each at face-centered-cubic (fcc) and hexagonallyclose-packed (hcp) three-fold hollow (3fh) sites $[7,8,17,18]$ (Figure 1b), or (iii) one each at bridge and $3 \mathrm{fh}$ sites $[19,20]$. Alternatively, it has been
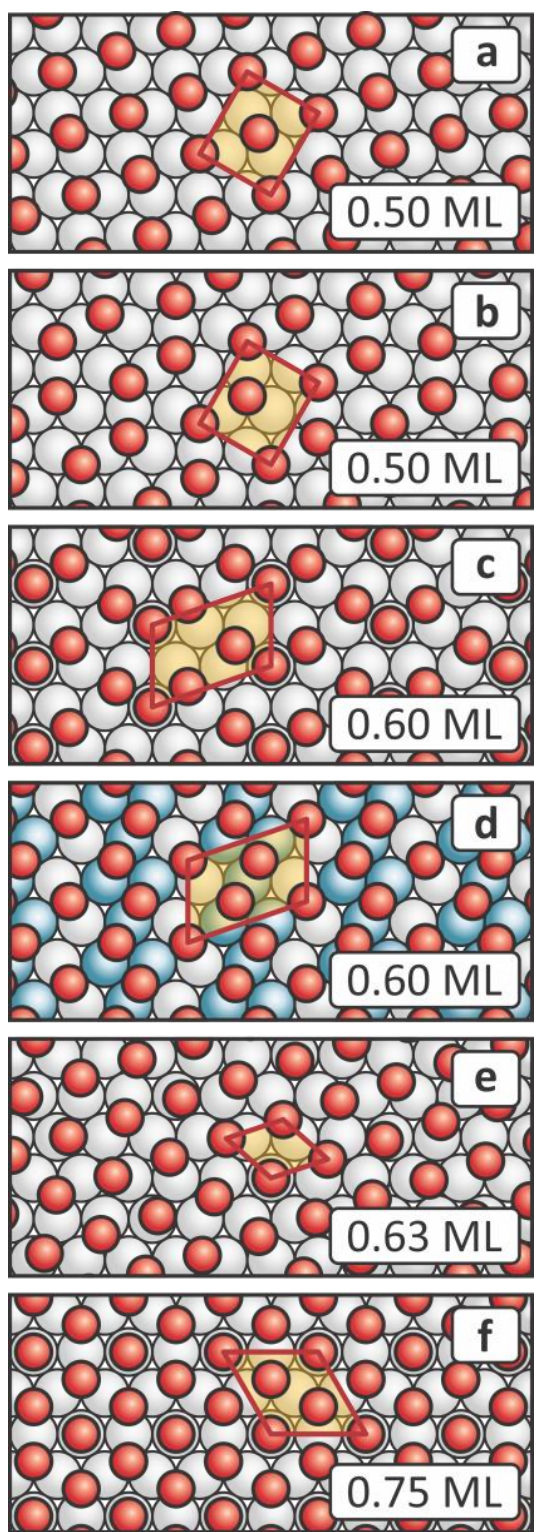

Figure 1. Models for selected $\mathrm{CO} / \mathrm{Pd}(111)$ structural phases. (a) Bridge-bonded and (b) hcp+fcc-bonded $c(4 \times 2)$ at $0.5 \mathrm{ML}$. (c) An early proposal for the $c(\sqrt{3} \times 5)$ rect phase at 0.6 ML. (d) The soliton model for the $c(\sqrt{3} \times 5)$ rect, with Pd atoms bound to the $\mathrm{COs}$ forming the heavy antiphase domain boundary tinted blue. (e) A compression structure proposed by Conrad et al., for 0.63 ML [3]; the unit mesh does not take the Pd into account. (f) The 3-CO $(2 \times 2)$ phase.

suggested that a mixture of exclusively-bridge and fcc-hcp domains coexist and interconvert at finite temperature [9]. A very recent density functional theory (DFT) study favors the fcc-hcp 
structure, argues against exclusive bridge-site occupation, and suggests an alternative structure where the COs adsorb between the fcc and bridge sites [11]. The adsorption energies of all of the proposed structures lie within $0.1 \mathrm{eV}$ (roughly 5\% of the total calculated adsorption energy) $[11,17,19,20]$. Because this relatively delicate energy balance may be influenced by surface strain [21], sub-surface impurities [9], and the presence of hydrogen [9], a full accounting for these extrinsic factors may need to be made before a final conclusion can be reached regarding CO site-occupation at 0.5 ML. Nevertheless, neither any of the 0.5-ML structures, nor the soliton models in the $0.5-0.6-\mathrm{ML}$ coverage range are consistent with the compression model.

The adsorption-site-geometry tendencies between 0.6 and 0.75 ML have drawn far less attention than those at $0.5-\mathrm{ML}$. One issue is the difficulty in growing well-ordered, fullysaturated $0.75-\mathrm{ML}$ adlayers with sharp LEED patterns. An early report indicated that the saturated surface was not well ordered [13], but more recent studies have found conditions under which reasonably sharp LEED spots are observed [22-24]. Based on evidence from IR [13,22], sum-frequency generation spectroscopy [24], STM [9,18] and DFT [9,17], it is now understood that at saturation, $\mathrm{CO}$ adopts a three-molecule-per-unit-mesh $(2 \times 2)$ lattice, with one $\mathrm{CO}$ each at a top, an fcc 3fh and an hcp 3fh site (Figure 1f). But adlayers between 0.6 and $0.75 \mathrm{ML}$ exhibit a series of at least six poorly-understood phases. Whether these phases can be explained in terms of the compression model or the soliton model remains to be determined. The large sizes of their unit meshes, and the corresponding large number of molecules per unit mesh, present a challenge to computer simulations of their packing geometries and diffraction intensities.

In this report we present LEED and temperature-programmed desorption (TPD) measurements made at and near saturation coverage. We identify a low temperature TPD feature whose full development is correlated with reaching saturation, and we describe a simple recipe 
for growing the fully-saturated adlayer. We identify a second TPD feature that is correlated with formation of a phase at $\sim 95 \%$ saturation coverage ( $\sim 0.71 \mathrm{ML})$. To the best of our knowledge this phase, which is a $\mathrm{c}(16 \times 2)$ adlayer net, has not been previously reported. By analogy with the bridge/near-bridge soliton models for structures in the $0.5-0.6 \mathrm{ML}$ range, we propose a molecular packing arrangement where strips with local 0.75 -ML coverage are separated by domain boundaries consisting of a vacancy row. We also report DFT calculations that identify a particular domain boundary structure and provide details regarding small displacements of the $\mathrm{CO}$ from high-symmetry surface sites. These displacements are largest near the domain boundaries. Thus this structure appears to lie at the boundary between a site-coincident phase, where the structure is dominated by molecule-substrate interactions, and a compression phase, where molecule-molecule lateral repulsion results in nearly hexagonally-packed adlayers that are only weakly commensurate with the substrate. The implications of this solution to the competition between interadsorbate repulsion and adsorption-site-specific interactions for other periodicities observed in the $0.6-0.6875 \mathrm{ML}$ coverage range are considered.

\section{Materials and Methods}

The experiments were conducted in an ion- and turbo-pumped ultrahigh vacuum (UHV) system (base pressure $<10^{-10}$ Torr) equipped with facilities for liquid-nitrogen cooling, ion bombardment, LEED, Auger spectrometry and TPD. Other experimental details have been published previously $[25,26]$.

Initial cleaning of the $\mathrm{Pd}(111)$ surface involved cycles of ion bombardment $\left(\mathrm{Ar}^{+}, 700 \mathrm{eV}\right.$, $1.2 \mu \mathrm{A}, 20 \mathrm{~min})$, annealing (1200 K) and oxidation (700-1100 K). For daily cleaning, oxidation

under $\mathrm{O}_{2}\left(p \sim 10^{-6}\right.$ Torr) during cycling between 770 and $1100 \mathrm{~K}$ at $2 \mathrm{~K} / \mathrm{s}$ was followed by 
flashing to $1000 \mathrm{~K}$. This resulted in sharp $(1 \times 1)$ LEED patterns, the absence of $\mathrm{CO}_{2}$ and $\mathrm{CO}$ signals below $\sim 800 \mathrm{~K}$ in TPD following oxygen exposure, and sharp $(2 \times 2)$ LEED patterns at saturation $\mathrm{CO}$ coverage as described below.

${ }^{12} \mathrm{C}^{18} \mathrm{O}$ was used in all experiments to avoid TPD signals from the ${ }^{12} \mathrm{C}^{16} \mathrm{O}$ background. The $\mathrm{CO}$ was introduced to the crystal surface through a retractable pinhole-type $(\sim 5 \mu \mathrm{m})$ gas doser, and the coverage could be precisely and reproducibly controlled by the pressure behind the doser pinhole, the exposure time, and the annealing temperature. TPD measurements were performed with a $2-\mathrm{K} / \mathrm{s}$ temperature ramp.

Periodic density functional theory calculations were performed using the Vienna Abinitio Simulation Package (VASP) [27-31] with the projector-augmented wave method [32]. The Pd substrate was modeled by periodically-repeated 64-atom 4-layer Pd slabs separated by $20 \AA$ of vacuum. The 16-Pd atom surface unit mesh was chosen to match the experimentally determined $\mathrm{c}(16 \times 2)$ surface lattice (see below). Atoms in the bottom $2 \mathrm{Pd}$ layers were fixed at their DFT-optimized bulk positions (calculated in the absence of $\mathrm{CO}$ ), while the top 2 Pd layers, as well as the $\mathrm{CO}$ admolecules, were fully relaxed. We have performed calculations using both the Perdew-Wang [33] and the Perdew-Burke-Ernzerhof [34] functionals within the generalized gradient approximation (GGA-PW91 and GGA-PBE, respectively) to describe exchangecorrelation effects. In the case of the PBE calculations, long-range electron correlation effects responsible for intermolecular dispersion interactions were included (Tkatchenko-Scheffler PBE+vdW scheme [35]) to gauge the significance of van der Waals forces. In both types of calculation, the cutoff energy was $500 \mathrm{eV}$, and convergences of the energy and forces were set to $1 \times 10^{-5} \mathrm{eV}$ and $0.01 \mathrm{eV} / \AA$, respectively. Monkhorst-Pack $k$-point sampling of the Brillouin zone [36] with $1 \times 5 \times 1$ meshes was used for the adlayer optimizations. 


\section{Experimental Results}

Figure 2 shows TPD measurements made for three $\mathrm{CO}$ adlayer preparations. The highest coverage adlayer was prepared by exposure to $\sim 2.0 \times 10^{-7}$ Torr $\mathrm{CO}$ for $6 \mathrm{~min}(\sim 72 \mathrm{~L})$ with the $\operatorname{Pd}(111)$ surface held at $135 \mathrm{~K}$, and is characterized by a well-developed desorption peak at $164 \mathrm{~K}$, in addition to peaks near 177, 230 and $460 \mathrm{~K}$, and a shoulder at $\sim 340 \mathrm{~K}$. The slightly lower-coverage adlayer in Figure 2 was prepared

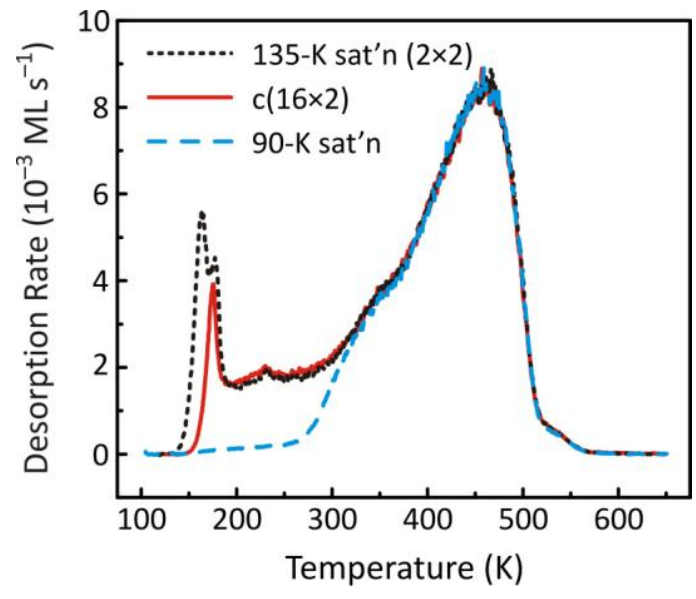

Figure 2. TPD of CO-saturated $\mathrm{Pd}(111)$ at $135 \mathrm{~K}$ before (short dashed black line) and after (solid red line) heating to $155 \mathrm{~K}$, and $\mathrm{CO}$-saturated $\mathrm{Pd}(111)$ at $90 \mathrm{~K}$ (long dashed blue line).

by heating the highest-coverage adlayer to $155 \mathrm{~K}$. This results in the disappearance of the 164-K desorption peak, but the 177-K feature, along with the other high-temperature features, remains. The integrated TPD yield for this lower-coverage adlayer is 0.95 times that for the highest coverage.

Figure 3 a shows the sharp $(2 \times 2)$ LEED pattern observed for the high-coverage adlayer in Figure 2. A (2×2) LEED pattern is expected for a CO-saturated $\operatorname{Pd}(111)$ surface [22-24,37]. Based on the high quality of the LEED pattern obtained, as well as the failure of all our attempts to further increase the coverage by varying the exposure pressure, time and temperature, we conclude that the highest-coverage TPD in Figure 2 corresponds to saturation coverage, and assign that coverage to be $0.75 \mathrm{ML}$ [13]. A correlation between formation of the $(2 \times 2)$ phase and desorption feature(s) in the 150-190-K range was reported previously [22,24]. However, we find the lowest-temperature feature most intense once full saturation is reached, whereas in previous reports the two features were either unresolved [22], or the lowest-temperature feature was 
relatively weak [24]. We associate the full development of the lowest-temperature TPD feature, namely the $164-\mathrm{K}$ peak, with completion of the saturation-coverage adlayer.

Consistent with previous reports $[20,22,37]$, we find that saturation was not reached by exposure to low $\mathrm{CO}$ partial pressures $\left(\sim 10^{-7}\right.$ Torr $)$ at low temperature $(\sim 90 \mathrm{~K})$. The lowest-coverage TPD shown in Figure 2 illustrates this behavior, showing that a saturation exposure $\left(\sim 1.3 \times 10^{-7}\right.$ Torr $\mathrm{CO}$ for $6 \mathrm{~min}, \sim 47 \mathrm{~L}$ ) at $90 \mathrm{~K}$ results in a coverage of $\sim 0.59 \mathrm{ML}$, well below that corresponding to the fully-saturated $(2 \times 2)$ structure, with no evidence of the low-temperature peaks in the TPD. That full saturation is attained only during growth at somewhat elevated temperature indicates that thermal activation assists the transition from the sub-saturation phase(s) to the saturated phase.
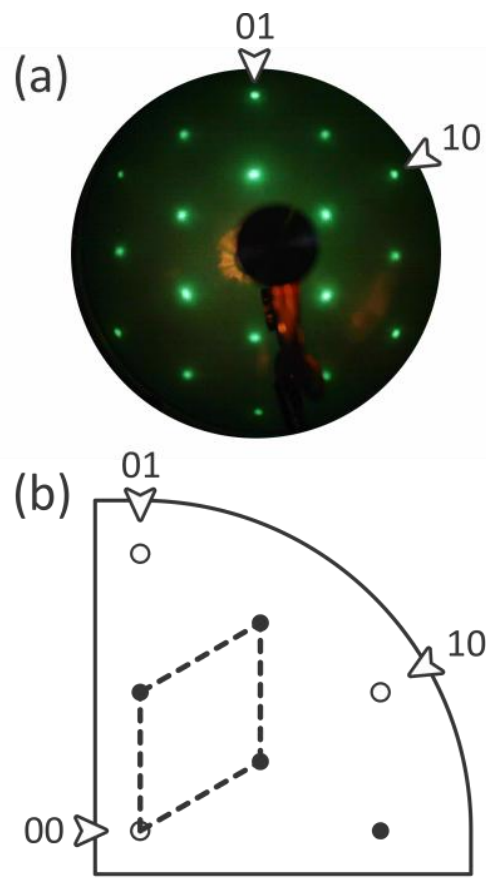

O $(1 \times 1)$

- $(2 \times 2)$

(c)

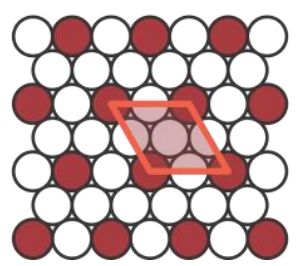

Figure 3. (a) A LEED pattern for $\mathrm{CO} / \mathrm{Pd}(111)$ after saturation at $135 \mathrm{~K}$, measured at a surface temperature of $90 \mathrm{~K}$ and electron energy of $64 \mathrm{eV}$. (b) The upper right-hand quadrant of reciprocal space with substrate diffraction indexed as indicated. The $(2 \times 2)$ adlayer unit mesh is indicated by dashed lines. (c) The corresponding real space $(2 \times 2)$ adlayer net with the unit mesh indicated. For the purpose of comparison with the net in Figure 4, filled circles indicate only the surface adlayer net, not the $\mathrm{CO}$ molecules ( $c f$. Figures $1 \mathrm{f}$ and 5).

In the previous reports it has been argued that nonequilibrium adsorption configurations, i.e., domains of coexisting sub-saturation coverage phases, can be "frozen in" at low temperatures, preventing additional adsorption at low temperature $[22,37,38]$. Our observation that saturation is not reached during $\sim 10^{-7}$ Torr $\mathrm{CO}$ exposure at $90 \mathrm{~K}$, as shown in Figure 2, 
supports this conclusion. Previous reports indicated that formation of a well-ordered $(2 \times 2)$ adlayer required heating (to 150 [20], 210 [37] or $600 \mathrm{~K}$ [22]) followed by cooling to $\sim 90$ $100 \mathrm{~K}$ during $\mathrm{CO}$ exposure. Also, the need for a high CO impingement rate (pressure $\sim 10^{-6}$ Torr CO) was suggested [22,37]. However, we find that a well-ordered $(2 \times 2)$ saturated adlayer phase can be grown at lower pressure and without cycling to high temperature, provided that the temperature is held at $\sim 135 \mathrm{~K}$ during growth.

Figure $4 \mathrm{a}$ shows the LEED pattern observed after heating the saturated adlayer to $155 \mathrm{~K}$ to remove the $164-\mathrm{K}$ feature (Figure 2). This pattern exhibits a splitting of the intensity in the vicinity of the $(2 \times 2)$ half-order diffraction spots. Specifically, in Figure $4 \mathrm{~b}$ the intensity near the $(0,1 / 2)$ and $(1 / 2,0)$ spots $(c f$. Figure $3 b)$ is split into five spots: a center spot remaining at the half-order position and four additional spots, two each lying along directions $\pm 120^{\circ}$ from the Pd next-nearest neighbor direction. The measured ratio of twice the average distance

Figure 4. (a) A LEED pattern for CO/Pd(111) after heating a saturation-coverage adlayer to $155 \mathrm{~K}$, measured at a surface temperature of $90 \mathrm{~K}$ and electron energy of $64 \mathrm{eV}$. (b) The upper right-hand quadrant of reciprocal space showing diffraction spots from the substrate, $(2 \times 2)$ mesh, and all three possible orientations of the $c(16 \times 2)$ mesh. One of the $c(16 \times 2)$ unit meshes is indicated by dashed lines. (c) The corresponding real space $c(16 \times 2)$ adlayer net with primitive unit mesh 1 indicated. The remainder of the centered unit mesh is shaded gray. Alternative equal-area unit mesh $\mathbf{2}$ is also indicated. For the purpose of comparison with the net in Figure 4, filled circles indicate only the surface adlayer net, not the $\mathrm{CO}$ molecules ( $c f$. Figure 5). 
between the six half-order spots, e.g., $(1 / 2,0)$ and $(0,1 / 2)$, to the average distance of the four satellite spots from the central half-order position is $16.8 \pm 0.9$. This splitting is consistent, within uncertainty, with a 16-fold periodicity along the Pd next-nearest neighbor direction ( [11 $\overline{2}]$ ). We find that the smallest unit mesh consistent with the observed pattern corresponds to a $c(16 \times 2)$ lattice, as illustrated in reciprocal and real space in Figure $4 \mathrm{~b}$ and $4 \mathrm{c}$, respectively. The adlayer consists of rows of some structural motif (discussed below) separated by a distance equal to $4 \sqrt{3} d_{\mathrm{Pd}}$ perpendicular to the rows and $2 d_{\mathrm{Pd}}$ along the rows. The $2 d_{\mathrm{Pd}}$ periodicity of neighboring rows is phase shifted by $\pi$. (A zero phase shift would correspond to an $(8 \times 2)$ lattice.) From the integrated area of the TPD we estimate a coverage of $0.71 \pm 0.04 \mathrm{ML}$, referenced to $0.75 \mathrm{ML}$ saturation coverage, for the $\mathrm{c}(16 \times 2)$ structure. This phase of $\mathrm{CO} / \mathrm{Pd}(111)$ has not, to the best of our knowledge, been reported previously. Development of this distinct structural phase is correlated with appearance of the 164-K TPD feature (Figure 2).

\section{Structural Analysis}

Based on the experimentally-determined coverage and unit mesh, we propose six working models for the molecular arrangement in the $c(16 \times 2)$ phase. These are shown in Figure 5, and the site occupation distributions are described in Table I. The rationale behind these models is as follows.

First, we infer that there must be $11 \mathrm{CO}$ molecules per unit mesh. The $\mathrm{c}(16 \times 2)$ unit mesh contains $16 \mathrm{Pd}$ atoms, thus there are only three possibilities: 10,11 , or 12 molecules per unit mesh, corresponding to coverages of $0.625,0.6875$, and $0.75 \mathrm{ML}$. The 11-molecule unit mesh is the only one that is in agreement, within the estimated uncertainty, with the experimentally 
determined coverage $(0.71 \pm 0.04 \mathrm{ML})$. And, we can confidently rule out 10 -and 12 -molecule unit meshes as possibilities. The former corresponds to a coverage well below the experimental value, and is in a coverage range where other structures with distinct LEED patterns have been observed by us and previously identified by others $[6,13,15]$. The latter corresponds to a coverage of $0.75 \mathrm{ML}$, the saturation coverage $(2 \times 2)$ adlayer prepared as described above and identified by others [22-24,37].

Second, because the coverage for the $\mathrm{c}(16 \times 2)$ phase is close to saturation, with little free surface area available, it is reasonable to construct a model for the $\mathrm{c}(16 \times 2)$ phase by beginning with the molecular arrangement in the $(2 \times 2)$ phase (see Figure 5) and removing one molecule in 12 . We find that this is easily achieved by introducing an antiphase domain boundary between strips of $(2 \times 2)$-ordered regions. This lowers the coverage from 12 to $11 \mathrm{CO}$ molecules per $16 \mathrm{Pd}$ atoms in the $\mathrm{c}(16 \times 2)$ unit mesh while maintaining the (2×2) 3-CO binding motif. This model is also consistent with reports showing bands at $\sim 2100$ and $1900 \mathrm{~cm}^{-1}$ in IR absorption [6,13] and SFG [23,24] at
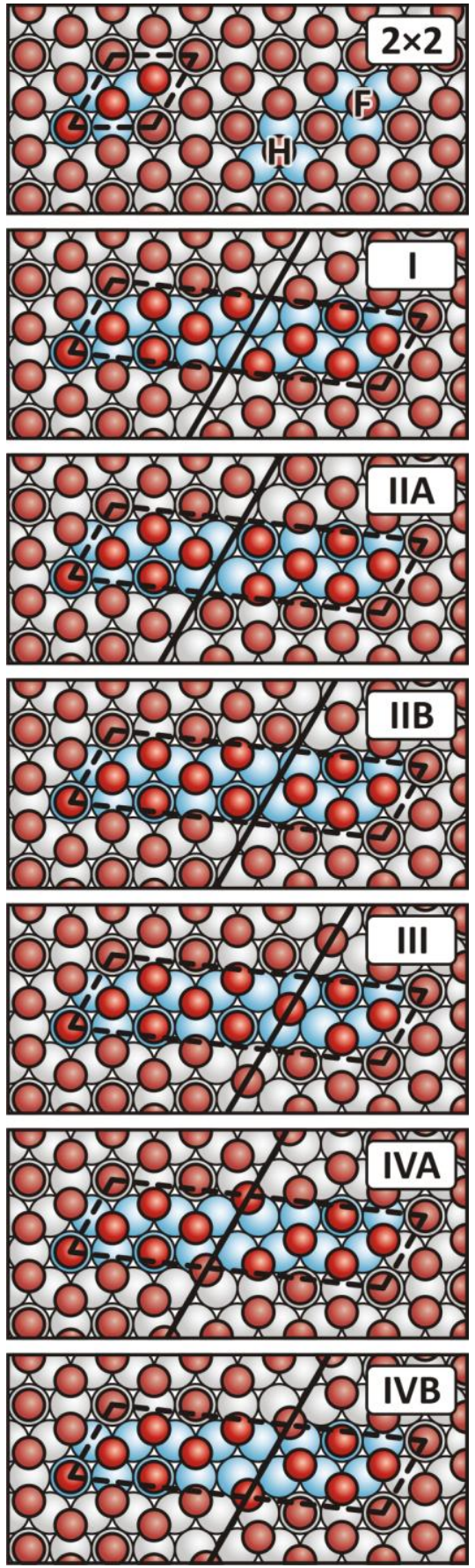

Figure 5. (2×2)-CO/Pd(111) (top) and six periodic anti-phase domain boundary models for $\mathrm{c}(16 \times 2)-\mathrm{CO} / \mathrm{Pd}(111)$. Unit meshes (dashed lines) and domain boundaries (solid lines) are indicated. F and $\mathrm{H}$ mark fcc and hcp sites, respectively. 
coverages just below saturation, indicating population of both top and 3fh sites. (Persistence of absorptions in the $1950-2000-\mathrm{cm}^{-1}$ region in some of these spectra suggests bridge-site occupation and would be consistent with coexistence of phases with differing local coverages as concluded in Ref. [24], or lower-coverage compression-model type structures, vide infra.)

\section{Table I}

Population distributions and the vacancy (Models I, IIA, IIB) or occupancy (Models III, IVA, IVB) types defining the domain boundaries in the six structural models prior to DFT optimization.

\begin{tabular}{ccccccc}
\hline \multirow{2}{*}{ site } & \multicolumn{6}{c}{ model } \\
& I & IIA & IIB & III & IVA & IVB \\
\hline \hline top & 3 & 4 & 4 & 4 & 3 & 3 \\
fcc 3-fold & 4 & 4 & 3 & 3 & 4 & 3 \\
hcp 3-fold & 4 & 3 & 4 & 3 & 3 & 4 \\
bridge & 0 & 0 & 0 & 1 & 1 & 1 \\
domain & top & hcp & fcc & bridge & bridge & bridge \\
boundary & & & & & & \\
\hline
\end{tabular}

Models I, IIA and IIB are all the possible variations on this theme, where the domain boundary is centered on top sites (Model I) or 3fh sites (Models IIA and IIB). Models IIA and IIB differ only in that their boundaries are centered over hcp and fcc $3 \mathrm{fh}$ sites, respectively. Models III, IVA and IVB are alternative structures where molecules near the domain boundary are repositioned onto bridge sites in an attempt to relieve compressive stress expected in the $(2 \times 2)$ structure where the $\mathrm{CO}$ molecules are packed more tightly than their van der Waals radii would be expected to permit [16]. Model III is the only case where this goal is achieved, but Models IVA and IVB are included for completeness. Only Models I and III possess glide or 
mirror plane symmetry with respect to the domain boundary that would allow for the possibility of the introduction of symmetric displacements out of the high-symmetry sites, and symmetric molecular tilting, with respect to the boundary, to relieve stress. Thus, in the absence of further information, we would favor these models as being most likely to accurately describe the molecular ordering in the $\mathrm{c}(16 \times 2)$ adlayer.

To determine if a minimum energy configuration exists, we used these six models as initial configurations and optimized their structures by minimizing their energies in DFT calculations. Using the PW91 functional, we found that optimization drives all six models to the same structure: essentially that labeled Model I in Figure 5. Supplementary calculations that accounted for interactions due to intermolecular electron correlations using the PBE+vdW functional were performed starting with Model I and Model III as initial configurations. The optimized PBE+vdW Model I structure was essentially identical to that found using the PW91 functional. Likewise, structural optimization using PBE+vdW drove Model III to the same configuration as that found when starting from Model I. We propose, then, that Model I is a good description of the molecular ordering within the $\mathrm{c}(16 \times 2)$ phase. The adsorption energy per $\mathrm{CO}$ extracted from the PW91 (PBE+vdW) calculations is $-1.53 \mathrm{eV}(-1.78 \mathrm{eV})$. Atomic coordinates for the minimum energy structure, along with illustrations of the corresponding adlayer viewed along the principal crystallographic axes, for both the PW91 and PBE+vdW calculations, are provided in the Supplementary Material.

The DFT calculations indicate that in the minimum-energy structure some of the molecules are displaced from the high-symmetry $3 \mathrm{fh}$ and top site positions. The calculated (PW91) lateral displacements of the carbon atoms are listed in Table II and illustrated in Figure 6. The predicted magnitude of the displacements ranges from essentially zero to $\sim 0.2 \AA$, 
approximately $6 \%$ of the $\mathrm{CO}-\mathrm{CO}$ nearest neighbor

distance. As we anticipated, based on the crowding in the $(2 \times 2)$ structure, the molecules are laterally displaced toward the domain boundary to relieve steric strain in the tightly-packed 3-CO arrangement. The displacements are largest for the COs nearest the domain boundary, and are symmetrical with respect to the boundary. We also find that some of the COs are tilted slightly away from the surface normal. Molecules 4, 6, 7 and 9 experience the largest tilts, which are on average only $2.5^{\circ}$ towards the domain boundary. All other tilts are less than $1^{\circ}$.

In addition, the surface $\mathrm{Pd}$ atoms are also displaced. The largest displacement is a $\sim 0.09 \AA$ downward shift of the two Pd atoms located on the
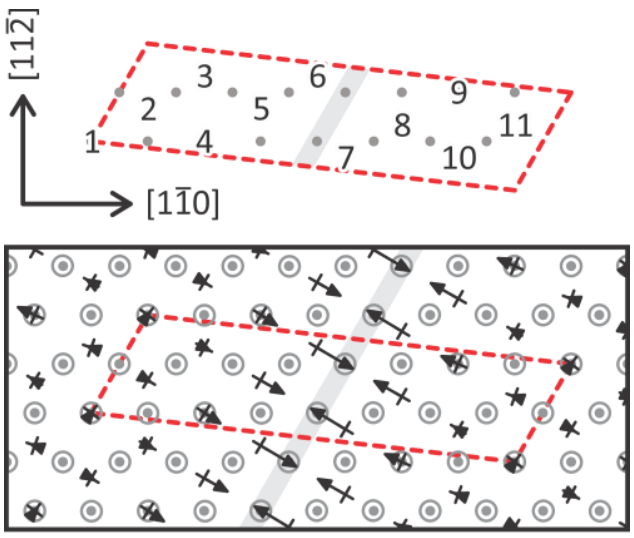

- Pdideal O Pd calc $\times$ Cideal

$\rightarrow$ C displacement $(\times 10)$

Figure 6. Calculated lateral displacements of the carbon atoms from their "ideal" highsymmetry adsorption sites. The arrows are the displacement vectors multiplied by 10 for visualization purposes. The crosses mark the corresponding high-symmetry sites. The $c(16 \times 2)$ unit mesh is indicated by dashed lines and the broad gray line marks the top-site vacancy domain boundary. Calculated displacement values are given in Table II, with $\mathrm{CO}$ molecules numbered as indicated in the legend above. Also shown are the calculated and ideal positions of the surface Pd atoms. The extremely small displacements (which are not multiplied by 10) are an order of magnitude smaller than those of the carbon atoms and are, therefore, nearly indiscernible in the Figure.

domain boundary with respect to the average height of the remaining 14 Pds. In addition, some of the Pd atoms are laterally displaced toward the boundary, albeit on a much smaller scale. There are six $\mathrm{Pd}$ atoms on either side of the domain boundary that comprise the $3 \mathrm{fh}$ sites at which CO molecules 5 and 8 are bound (see Figure 6). These are displaced $\sim 0.03 \AA$ on average toward the domain boundary, along the same direction that the $\mathrm{CO}$ molecules are displaced. The two Pd atoms on the boundary show very small $\sim 0.01 \AA$ displacements from the boundary, in a direction opposite to that of the $\mathrm{CO}$ molecules to which they are bound (numbered 6 and 7 in Figure 6). Overall, the preference for $\mathrm{CO}$ binding to high-symmetry sites predominates, but 
interadsorbate repulsion is significant, resulting in displacement of the adsorbates toward the domain boundary, in addition to a very small reconstruction of the surface Pd. The resultant structure represents a compromise between molecule-substrate and molecule-molecule interactions that largely favors the molecule-substrate interactions.

\section{Table II}

DFT-calculated (PW91) lateral displacements of the carbon atoms from the nearest top and $3 \mathrm{fh}$ sites. The molecule numbering scheme is given in Figure 6.

\begin{tabular}{ccccc}
\hline admolecule & site & $\begin{array}{c}\delta[1 \overline{1} 0] \\
(\AA)\end{array}$ & $\begin{array}{c}\delta[11 \overline{2}] \\
(\AA)\end{array}$ & $\begin{array}{l}\delta r \\
(\AA)\end{array}$ \\
\hline \hline 1 & top & 0.0103 & 0.0124 & 0.0161 \\
2 & fcc 3fh & 0.0137 & -0.0087 & 0.0162 \\
3 & hcp 3fh & 0.0267 & -0.0136 & 0.0300 \\
4 & top & 0.0793 & -0.0511 & 0.0944 \\
5 & fcc 3fh & 0.1148 & -0.0655 & 0.1322 \\
6 & hcp 3fh & 0.1751 & -0.1038 & 0.2035 \\
7 & fcc 3fh & -0.1695 & 0.1015 & 0.1975 \\
8 & hcp 3fh & -0.1347 & 0.0761 & 0.1547 \\
9 & top & -0.0832 & 0.0379 & 0.0914 \\
10 & fcc 3fh & -0.0223 & 0.0119 & 0.0253 \\
11 & hcp 3fh & -0.0169 & 0.0048 & 0.0175 \\
\hline
\end{tabular}

To provide a visual perspective on the interactions we evaluated the charge density difference distribution

$$
\Delta \rho(\boldsymbol{r})=\rho[\mathrm{CO} / \mathrm{Pd}(111)](\boldsymbol{r})-\sum_{i=1}^{i=11} \rho\left[\mathrm{CO}_{i}\right](\boldsymbol{r})-\rho[\operatorname{Pd}(111)](\mathbf{r})
$$

where $\rho[\mathrm{CO} / \mathrm{Pd}(111)](\boldsymbol{r}), \rho\left[\mathrm{CO}_{i}\right](\boldsymbol{r})$, and $\rho[\operatorname{Pd}(111)](\boldsymbol{r})$, are the charge densities for the full 11- 
$\mathrm{CO}$ c(16×2) / 4-layer $\mathrm{Pd}(111)$ slab unit cell, each of the 11 individual $\mathrm{CO}$ molecules, and the 4layer Pd slab, respectively, and $\boldsymbol{r}$ is the 3D position vector. All of the densities were calculated at the PBE+vdW level with the coordinates of the atoms frozen in the minimum energy configuration found by that method for the $\mathrm{c}(16 \times 2) \mathrm{CO} / \mathrm{Pd}(111)$ system.

Figure 7 shows charge density difference isosurfaces extracted from the full charge density difference calculation, with regions enclosed by charge-difference contours of $+(-) 0.00526$ electrons $/$ Bohr $^{3}$ shown in yellow (blue). These surfaces show that the most

Figure 7. The charge density difference isosurfaces described in the text viewed (a) down the surface normal and (b) along unit mesh vector $\boldsymbol{B}$. The ideal $2 \mathrm{D}$ unit meshes for the first, second and third layer Pd (red rhombuses), as well as the top-site vacancy domain boundary (broad red line), are indicated in (a). A close-up, tilted view centered on an fcc $\mathrm{CO}$ is shown in (c), where the three surface $\mathrm{Pd}$ atoms comprising the fcc 3 fh site are labeled "Pd1." Regions of increased (decreased) electron density are colored yellow (blue), with the orange (green) areas indicating truncation of the isosurface at the plane containing unit mesh vector $\boldsymbol{A}$ and the surface normal. The same three top-, fccand hcp-site COs are labeled "T," "F" and " $\mathrm{H}$," respectively, in all three panels. (a)

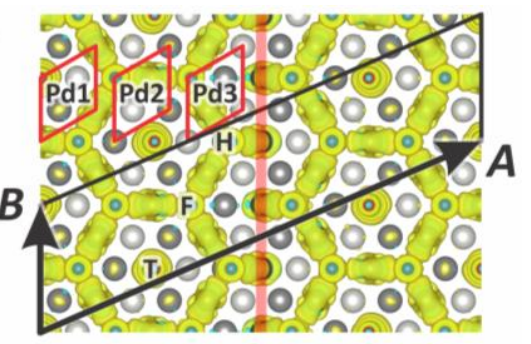

(b)

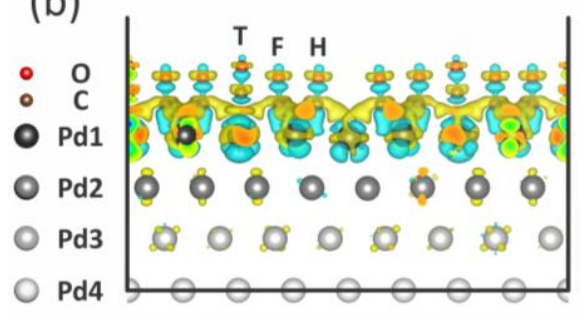

(c)

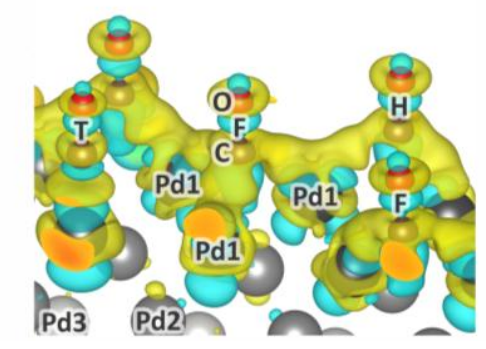

significant charge density increase due to adsorption is between the carbon atoms and the Pd atoms of the $3 \mathrm{fh}$ sites. The most significant decreases are observed around the Pd atoms in the top layer and directly beneath the $\mathrm{C}$ atoms. These charge density differences are consistent with donation from the $\mathrm{CO} 5 \sigma$ orbital (blue lobes beneath $3 \mathrm{fh} \mathrm{CO}$ in Figure 7) to the $\mathrm{Pd}$, "back- 
donation" from the out-of-plane Pd 5d orbitals (blue lobes on surface Pd atoms), presumably into the $\mathrm{CO} 2 \pi^{*}$ orbital, and formation of strong C-Pd bonds indicated by charge accumulation between the $\mathrm{C}$ and Pd atoms (3-fold yellow lobes at 3fh sites and yellow lobe between $\mathrm{C}$ and Pd at top sites). In an effort to visualize charge redistribution due only to the proximity of the COs, we calculated the charge density difference as in Equation 1, but with the Pd entirely removed from the calculation. Though an artificial construct, this allows us to gauge the scale of the molecule-molecule interactions in this closely-packed geometry. These calculations showed no significant charge redistribution, reinforcing the conclusion that the molecule-substrate interactions are predominant. This observation is also consistent with the result that inclusion of long-range intermolecular interactions using the PBE+vdW resulted in the same adlayer geometry as did the PW91, sans vdW, calculation. Thus, in determining the geometry of the $c(16 \times 2)$ phase, molecule-substrate interactions play the most significant role; the displacements of the COs indicate that intermolecular repulsion plays a secondary role; and the role of intermolecular attraction is negligible.

Finally, to investigate the possibility of a "compression-model" structure, and as a check of the uniqueness of the DFT-optimization, we devised an alternative model based on the continuous compression concept as a starting point for geometry optimization. This starting structure, depicted in Figure 8, was designed so that one $\mathrm{CO}$ is bound at a $3 \mathrm{fh}$ fcc site. We chose this 3 fh site as an initial "registry" point because it has been shown to be the most strongly binding $[9,39]$, thus we would expect the structure in Figure 8 to give this compression-model structure the best chance of reaching an energy minimum competitive with that of Model I. The remaining molecules in the unit mesh are located on a distorted hexagonal net with lattice parameters chosen such that the distorted hexagonal unit mesh is a sublattice of the $c(16 \times 2)$ net according to the equation: 


$$
\left(\begin{array}{l}
\boldsymbol{A} \\
\boldsymbol{B}
\end{array}\right)=\left(\begin{array}{cc}
3 & -4 \\
2 & 1
\end{array}\right)\left(\begin{array}{l}
\boldsymbol{a} \\
\boldsymbol{b}
\end{array}\right)
$$

where, as defined in Figure 8, the upper case letters, $\boldsymbol{A}$ and $\boldsymbol{B}$, are the $\mathrm{c}(16 \times 2)$ unit mesh vectors, and the lower case letters, $\boldsymbol{a}$, and $\boldsymbol{b}$ are the sublattice unit vectors. Thus, the CO were distributed within the $c(16 \times 2)$ unit mesh so as to maximize their separation without any preference for surface binding sites, aside from the choice of the initial registry point. Having made this attempt to devise a reasonable compression-model structure, we found that DFT geometry optimizations from this starting point using the PW91 and PBE+vdW functionals both resulted in migration of the $\mathrm{CO}$ to the same binding sites occupied in Model I. This gives further support to Model I as a good description of the molecular ordering in the $\mathrm{c}(16 \times 2)$ adlayer, and argues for a coincident-site lattice as opposed to a compression-model interpretation for this sub-saturation structure.

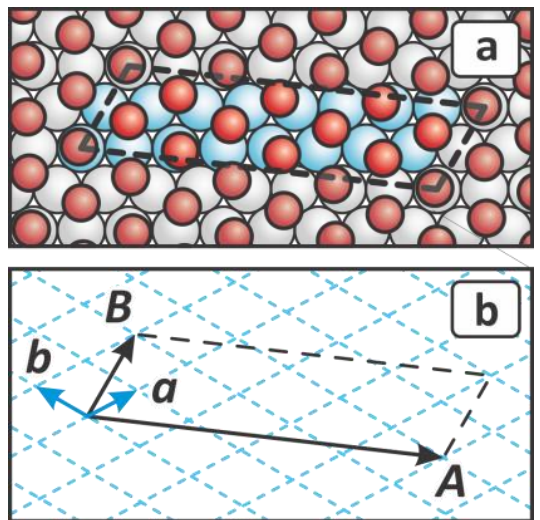

Figure 8. (a) Schematic of a compression structure consisting of a distorted hexagonal lattice. The molecular coordinates were chosen such that one molecule per $c(16 \times 2)$ unit mesh (dashed line) is bound to an fcc $3 \mathrm{fh}$ site (far left-hand side of the $c(16 \times 2)$ unit mesh). The remaining molecules are positioned on a distorted hexagonal net. (b) Scheme showing the relationship between the $\mathrm{c}(16 \times 2)$ unit mesh (vectors $\boldsymbol{A}$ and $\boldsymbol{B}$, black) and the distorted hexagonal net ( $\boldsymbol{a}$ and $\boldsymbol{b}$, blue).

\section{Discussion}

At least eight distinct $\mathrm{CO} / \mathrm{Pd}(111)$ adlayer structures have been reported to span the $0.6-$ 0.75-ML coverage range. These are compiled in Table III, with the $c(16 \times 2)$ structure included, placing it in the context of the coverage-dependent phase behavior of this system. With the exception of the first and last structures in this sequence, to the best of our knowledge, the 
detailed arrangements of the $\mathrm{CO}$ molecules in these lattices are not known. Thus the $(2 \times 2)$ antiphase domain-boundary model we propose for the $c(16 \times 2)$ represents a first step in understanding the structural phase transitions that link the $\mathrm{c}(\sqrt{3} \times 5)$ rect $0.6-\mathrm{ML}$ phase and the saturation $0.75-\mathrm{ML}(2 \times 2)$ phase. In the following we discuss the structures observed between 0.5 and $0.75 \mathrm{ML}$ in terms of two competing models: a "heavy" domain-boundary bridge-site model and our "light" domain-boundary $(2 \times 2)$ model, where the terms "heavy" and "light" indicate that the adsorbate density in the boundaries is higher or lower, respectively, than in the domains. We show that the low-coverage limits of both models predict the same periodicity at $0.5 \mathrm{ML}$, where the two types of structures have been proposed to coexist [9]. The system is believed to prefer the bridge-site model for coverages above 0.5 ML up to 0.6 ML [6], at which point it is no longer feasible (see below). The $(2 \times 2)$ model describes the ordering only at and approaching saturation, leaving a gap between 0.6 and $0.6875 \mathrm{ML}$ where we hypothesize that the ordering can be characterized as pseudo-commensurate compression-type structures.

To begin to understand the structural diversity, it is reasonable to look for unifying relationships among the structures. The soliton model proposed by Tüshaus, et al. [6], identified such a relationship among the progression of seven distinct structural phases that span the $0.5-$ 0.6-ML range. Tüshaus, et al., proposed, and more recent STM images support the idea [10], that the observed periodicities in this regime can be explained by an all bridge-site model where strips of the 2-CO all-bridge $\mathrm{c}(4 \times 2)$ phase with local $0.5-\mathrm{ML}$ coverage (see Figure $9 \mathrm{a}, c f$. Figure 1a), are separated by periodic heavy antiphase domain boundaries with a local coverage equivalent to a $(1 \times 1)$ adlayer. Small displacements of the $\mathrm{CO}$ off the bridge sites away from the domain boundary were posited to reduce repulsive adsorbate interactions [6], analogous to those predicted by our DFT calculations for the $c(16 \times 2)$ structure. An example, the $c(\sqrt{3} \times 9)$ rect 
adlayer structure is shown in Figure $9 b$. The ordering within the $c(4 \times 2)$ strips can be thought of as a herringbone pattern, where the fundamental unit is two $\mathrm{Pd}$ atoms and the $\mathrm{CO}$ molecule bridge-bonded between them, as indicated in Figure 9a. Two of these units comprise the primitive $c(4 \times 2)$ unit mesh. The series of structures in the $0.5-0.6-\mathrm{ML}$ range are $(\sqrt{3} \times[2 m+1])$ rect $\quad$ for $\quad$ odd $\quad m$ and $c(\sqrt{3} \times[2 m+1])$ rect for even $m$, where $m$ is twice the number of $c(4 \times 2)$ units between the domain boundaries. This soliton model is a compelling explanation for the observed complex sequence of LEED patterns and IR spectra; LEED patterns for $m=2,3,4,5,8$ and 17 have been reported [6]. For the purpose of our discussion, we note that $m=2$, where there is only one $c(4 \times 2)$ unit between the domain boundaries (see Figure 1d), corresponds to the $0.6-\mathrm{ML} c(\sqrt{3} \times 5)$ rect structure listed in Table III.
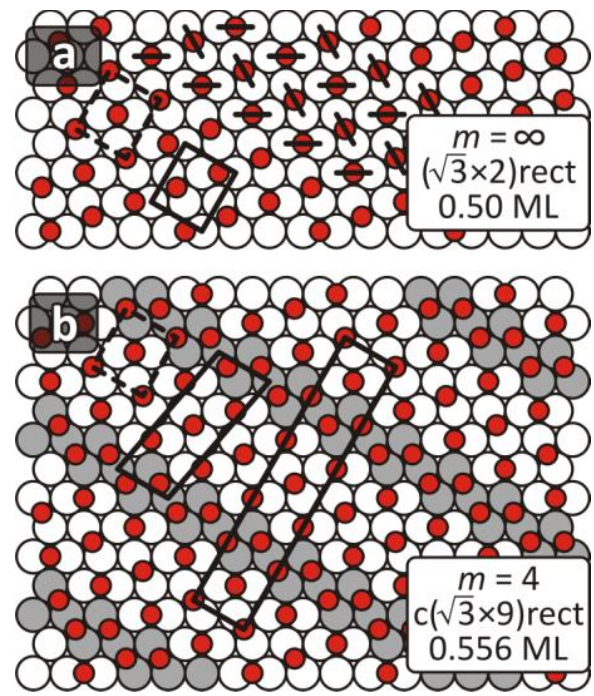

Figure 9. Bridge-site models for the (a) $(\sqrt{3} \times 2)$ rect, i.e., $\mathrm{c}(4 \times 2)$, and (b) $\mathrm{c}(\sqrt{3} \times 9)$ rect adlayers. The fundamental unit, the $c(4 \times 2)$ primitive unit mesh, is indicated by dashed lines. In (a) the solid-line shows an alternative $c(4 \times 2)$ unit mesh to aid comparison with the 3 fh-site structure in Figure 10, and the herringbone ordering of the bridge bonding is indicated in part of the figure. In (b) Pd atoms in the heavy domain boundaries are shaded gray and both primitive and centered $c(\sqrt{3} \times 9)$ rect unit meshes are indicated. For simplicity, we have not included the expected displacements of the $\mathrm{CO}$ in the domain boundary regions.

\section{Table III}

$\mathrm{CO} / \mathrm{Pd}(111)$ structures reported for the coverage range 0.6-0.75 ML. The number of $\mathrm{Pd}$ atoms and $\mathrm{CO}$ molecules per primitive unit mesh and the corresponding coverage, $N_{\mathrm{Pd}}, N_{\mathrm{CO}}, \theta_{\text {nom }}$, respectively, are given. The value of $d_{\mathrm{Pd}} / \sqrt{\theta}$ is the intermolecular spacing based on the unit mesh area and coverage assuming hexagonal packing, and is provided as a point of comparison with the van der Waals diameter of carbon, 3.4 $\AA$. In addition to the structures listed, two others were 
observed in STM by Rose et al., with periodicities close to $\sqrt{7} d_{\mathrm{Pd}}$ and $5 d_{\mathrm{Pd}}$, but the unit mesh orientation, coverage, and molecular packing for these structures were not specified in that work [9].

\begin{tabular}{|c|c|c|c|c|c|c|c|}
\hline $\begin{array}{c}\theta_{\text {nom }} \\
(\mathrm{ML})\end{array}$ & $\begin{array}{l}\text { proposed } \\
\text { lattice }\end{array}$ & Ref. & $\begin{array}{c}a \\
\left(d_{\mathrm{Pd}}\right)\end{array}$ & $\begin{array}{c}b \\
\left(d_{\mathrm{Pd}}\right)\end{array}$ & $N_{\mathrm{Pd}}$ & $N_{\mathrm{CO}}{ }^{*}$ & $\frac{d_{\mathrm{Pd}}}{\sqrt{\theta}}$ \\
\hline 0.6000 & $\mathrm{c}(\sqrt{3} \times 5) \mathrm{rect}$ & {$[6,9,15]$} & $\sqrt{3}$ & $\sqrt{7}$ & 5 & 3 & 3.550 \\
\hline 0.6094 & $(4 \sqrt{3} \times 8)$ rect & {$[6,15]$} & $4 \sqrt{3}$ & 8 & 64 & 39 & 3.523 \\
\hline 0.6250 & $\left(\begin{array}{rr}4 & -2 \\
2 & 3\end{array}\right)$ & {$[6]$} & $\sqrt{7}$ & $2 \sqrt{7}$ & 16 & 10 & 3.479 \\
\hline 0.6364 & $(3 \sqrt{3} \times 11) \mathrm{rect}$ & {$[6]$} & $3 \sqrt{3}$ & 11 & 66 & 42 & 3.465 \\
\hline $0.6765^{\dagger}$ & “split $(2 \times 2) "\left(\begin{array}{rr}17 & 17 \\
8 & 16\end{array}\right)$ & {$[8,13,15]$} & $8 \sqrt{3}$ & 17 & 136 & 92 & 3.344 \\
\hline 0.6875 & $c(16 \times 2)$ & this work & 2 & $7 *$ & 16 & 11 & 3.317 \\
\hline 0.7500 & $(2 \times 2)$ & {$[6,8,9,13,15]$} & 2 & 2 & 4 & 3 & 3.175 \\
\hline
\end{tabular}

* Determination of the number of $\mathrm{CO}$ molecules per unit mesh for selected adlayers is discussed in the Supplementary Material.

$\dagger$ Coverage based on a hypothetical unit mesh and structure proposed in Ref [15]. That same work reports observation of a split $(2 \times 2)$ structure with decreasing splitting at unspecified coverages less than full saturation.

$\$$ At least two reasonable primitive unit meshes for the $\mathrm{c}(16 \times 2)$ net can be conceived: $1: a=2 d_{\mathrm{Pd}}, b=\sqrt{57} d_{\mathrm{Pd}}$, $\alpha=113.41^{\circ}$ and 2: $a=2 d_{\mathrm{Pd}}, b=7 d_{\mathrm{Pd}}, \alpha=98.21^{\circ}$ (refer to Figure 4). We list $\mathbf{2}$ in the table because it has the shortest lattice parameters. We have used $\mathbf{1}$ in the figures because it has a clearer relationship to the conventional centered $\mathrm{c}(16 \times 2)$ unit mesh. 
By analogy to the bridge-site heavy domain-boundary model, in Figure 10 we show possible structures derived from our light domain-boundary model for the $c(16 \times 2)$ adlayer. Given strips with local 3-CO $(2 \times 2)$ 0.75-ML ordering separated by periodic top-site vacancy domain boundaries of the type in Model I (Figure 5), we find a series of structures that can be described as $c(4 n \times 2)$, where $n$ is the number of unit $(2 \times 2)$ meshes that would occupy the distance between vacancy rows were the adlayer fully saturated. Only two such structures can be built between $0.6875(n=4)$ and $0.6 \mathrm{ML}$, viz., $n=3$ and $n=2$, corresponding to 0.6667 and 0.625 ML, respectively. However, the resultant $\mathrm{c}(12 \times 2)$ and $\mathrm{c}(8 \times 2)$ lattices do not correspond to any of the reported structures listed in Table III, indicating that the light domain-boundary model does not explain the progression of structures in the $0.6-0.75-\mathrm{ML}$ range.

Nevertheless, this exercise provides insight into the ordering of $\mathrm{CO}$ on $\mathrm{Pd}(111)$. Notably, when the light-domain boundary model is extended to the lowest possible coverage, i.e., $n=1$, it predicts an alternative 2-CO $\mathrm{c}(4 \times 2) \quad 0.5 \mathrm{ML}$ structure, with $\mathrm{CO}$ bound to fcc and hcp 3fh sites (Figure 10d, $c f$. Figure 9a and 1b). Based on STM images,
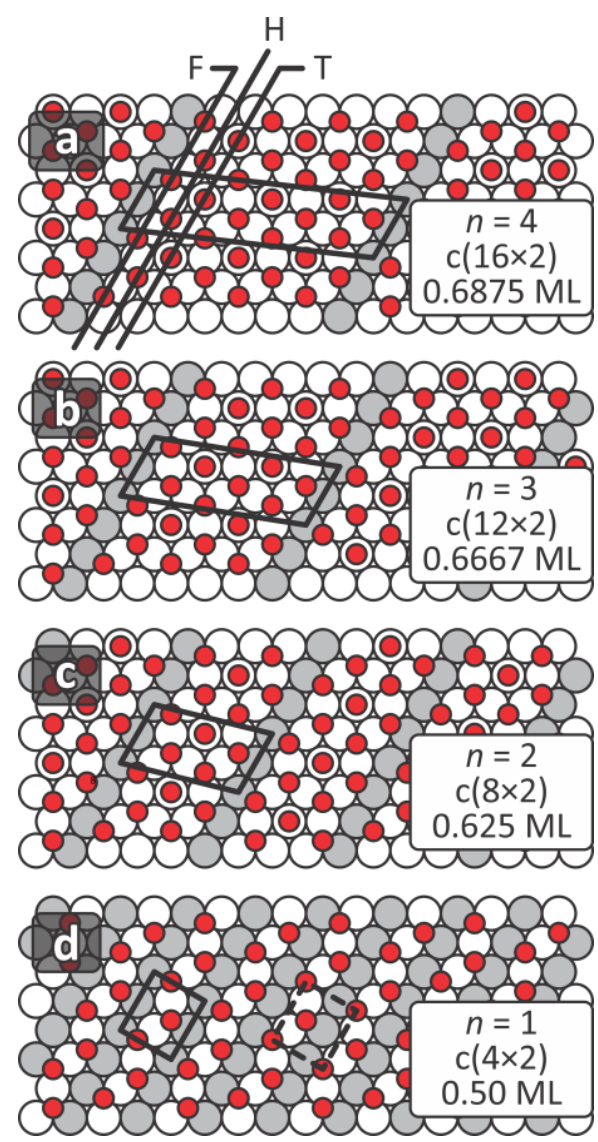

Figure 10. Structures that can be built from the 3-CO $(2 \times 2)+$ top-site vacancy domain boundary model. Pd atoms at the domain boundaries are shaded gray. Rows of $\mathrm{CO}$ bound to fcc, hcp and top sites are indicated by lines labeled "F," "H," and "T," respectively. Primitive unit meshes are indicated by solid lines, and the dashed line shows an alternative choice for the $\mathrm{c}(4 \times 2)$, for comparison to the bridgesite structure in Figure 9. 
Rose et al., concluded that this fcc-hcp structure coexists with the bridge-site $2-\mathrm{CO}$ c $(4 \times 2)$ structure (Figure 9a), with the latter occurring slightly more frequently at $120 \mathrm{~K}$ [9]. Analyses of high-resolution core level photoemission spectra [8] and photoelectron diffraction data [7], as well as recent DFT calculations [11] argue for the conclusion that the fcc-hcp structure is the best, and possibly true, explanation for the $\mathrm{c}(4 \times 2)$ ordering. Considering that there is now evidence supporting both the heavy-domain-boundary [10] and the light domain-domainboundary models (this work) at coverages $>0.5 \mathrm{ML}$, we propose that at $0.5 \mathrm{ML}$ the system is at a "tipping point" between these two soliton-related models. We note that a $\sim 0.01-\mathrm{ML}$ increase in coverage causes the system to apparently abandon the $3 \mathrm{fh}$ binding altogether in favor of the $(1 \times 1)$ bridge-site soliton model, as signaled by the appearance of $(\sqrt{3} \times 35)$ rect ordering at 0.514 ML [6], which cannot be explained by our $(2 \times 2)$ light domain-boundary model.

A key feature of the heavy domain-boundary solitons in comparison to the $(2 \times 2)$ light domain-boundary model ( $c f$. Figures $9 \mathrm{a}$ and 10d), is that they sacrifice 3 fh-site binding in favor of minimizing the number of $\mathrm{CO}$ molecules that share a single Pd atom. This suggests - and the small $\sim 0.1 \mathrm{eV}$ difference in adsorption energies between the two $0.5 \mathrm{ML} \mathrm{c}(4 \times 2)$ structures $[11,17,19,20]$ supports - the idea that though the 3 fh sites bind CO more strongly than bridge sites [39], at 0.5 ML the free energy decrease associated with separating the two CO so that they do not share a single Pd atom (as they do in the $3 \mathrm{fh}$ structure, Figure 10d) is nearly, but not quite completely, offset by the free energy increase associated with binding to bridge sites (Figure 9a) rather than $3 \mathrm{fh}$ sites (Figure 10d).

With increasing coverage beyond $0.6 \mathrm{ML}$, however, we expect the heavy domainboundary model to no longer be energetically favorable. This is because at 0.6 ML $(m=2)$ the regions of $(1 \times 1)$ packing are separated by only a single $\mathrm{c}(4 \times 2)$ unit, and to exceed $0.6 \mathrm{ML}$, the 
bridge-type solitons would collide, resulting in extended areas of highly compressed $(1 \times 1)$ packing. Thus, at this coverage we would expect the $3-\mathrm{CO}(2 \times 2)$ packing to be preferred. However, the periodicities predicted by the $3-\mathrm{CO}(2 \times 2)$ model at coverages above $0.6 \mathrm{ML}$, i.e., $n=2$ and 3 (Figure 10b and c), are not observed until $0.6875 \mathrm{ML}(n=4)$. Instead, there is a progression of ordered structural phases with large unit meshes (Table III) for which we find no relationship with the $3-\mathrm{CO}(2 \times 2)$ packing. In this coverage regime $(0.6 \mathrm{ML}<\theta<0.6875 \mathrm{ML})$ the $\mathrm{CO}$ density is sufficiently high that the number of ways of arranging the $\mathrm{CO}$ in the observed unit cells (Table III), under the constraint that they be at (or near) high symmetry sites, is quite limited. Only local $3-\mathrm{CO}(2 \times 2)$ or bridge-site (or other site) $(1 \times 1)$ structures have high enough densities to be considered. Because both of these models fail to predict the observe periodicities, we speculate that true compression structures, comprised of molecules arrayed in a distorted hexagonal lattice that has only long-range commensurability, may characterize the ordering in this narrow coverage range. In such structures, the interactions among the $\mathrm{CO}$ would ultimately determine the local ordering, resulting in significant displacements from high-symmetry sites, similar to that in Figure 8a. However, because they include molecules that are near top and bridge sites, they are consistent with the observation of bridge and top-site absorptions in IR spectra at coverages above 0.6 ML $[6,13,24]$. DFT calculations, as well as molecular dynamics simulations of adlayers in this coverage range are needed to explore this possibility.

Our results indicate that at $0.6875 \mathrm{ML}$ the decrease in free energy associated with binding two-thirds of the $\mathrm{CO}$ at $3 \mathrm{fh}$ sites offsets the increase in repulsive interaction associated with packing the molecules somewhat more tightly than they would be in a distorted hexagonal array. That the $c(12 \times 2)$ and $c(8 \times 2)$ structures are not observed suggests that this tradeoff does not become favorable until the coverage is very near saturation. The well-developed TPD feature 
associated with the $\mathrm{c}(16 \times 2)$ structure (Figure 2) appears to mark this significant shift in the energy balance at this coverage. The coverage where this shift occurs, $\sim 0.66 \mathrm{ML}$, matches closely that at which the intermolecular distance (Table III) drops below the $3.4 \AA$ van der Waals diameter of carbon [16].

Regarding the difficulties in growing high-coverage adlayers at low temperature, we note that the $0.59-\mathrm{ML}$ saturation coverage we observe at a growth temperature of $\sim 90 \mathrm{~K}$ (Figure 2) coincides with what we propose is a transition from the soliton-type order to the distortedhexagonal order regime. At this coverage, the molecules are nearly close-packed; additional adsorption would require thermal fluctuations and involve reorganization over a large length scale to provide incident $\mathrm{CO}$ access to the substrate. Thus it seems reasonable to expect this to result in a kinetic limitation to achieving saturation at low temperature. Further investigation of the temperature and pressure dependences of the adlayer growth and phase behavior would permit identification and quantification of this and other possible kinetic traps as well as possible non-equilibrium structures.

Finally, we note that we cannot rule out the formation of longer-range commensurate structures such as $\mathrm{c}(20 \times 2)$, i.e., $n=5$, or higher. Such lattices may be a more reasonable explanation for the reported "split" $(2 \times 2)$ LEED patterns, whose symmetry and coverage as listed in Table III are speculative [15]. STM observations of disordered domain boundaries between local $(2 \times 2)$ ordering at coverages approaching saturation suggest that the energetic difference between ordered and disordered domain walls may be vanishingly small as the coverage approaches saturation, such that well-ordered large unit mesh structures may be difficult to prepare. Annealing and cooling to lower temperatures ( $<90 \mathrm{~K})$ should be investigated before reaching such a conclusion. 


\section{Conclusions}

Temperature programmed desorption, low energy electron diffraction, and density functional theory calculations have been used to investigate the molecular packing of $\mathrm{CO}$ adlayers on $\operatorname{Pd}(111)$ at a coverage just below saturation. We have correlated two distinct lowtemperature features in the TPD measurement made after fully saturating the surface at $135 \mathrm{~K}$ with saturation and slightly sub-saturation phases at 0.75 and 0.6875 ML. Annealing the fully saturated adlayer to $155 \mathrm{~K}$ produces a well-ordered sub-saturation phase whose LEED pattern we have assigned as a $c(16 \times 2)$ lattice. DFT calculations support a model for ordering in this adlayer that involves adsorption at, or very near, high-symmetry sites. Specifically, we find that the molecules form strips with a local $(2 \times 2)$ ordering separated by periodic light antiphase domain boundaries. There are eleven $\mathrm{CO}$ molecules in the primitive unit mesh, four adsorbed at fcc $3 \mathrm{fh}$ sites, four at hcp $3 \mathrm{fh}$ sites, and three at top sites. The domain boundary consists of top-site vacancies.

Comparison of the $c(16 \times 2)$ with other CO structures that have been observed on $\operatorname{Pd}(111)$ at coverages of $0.5 \mathrm{ML}$ and above suggests that the $(2 \times 2)+$ top-site vacancy motif is energetically competitive with bridge-site + heavy antiphase domain boundary structures at $0.5 \mathrm{ML}$. The bridge-site structures dominate the phase diagram from $>0.5$ to $0.6 \mathrm{ML}$, but above 0.6 ML and below 0.6875 ML, neither of these ordering motifs explain reported observations. We hypothesize that the ordering in this coverage range may be best described as exhibiting long-range commensurability where the molecules assume distorted hexagonal packing to minimize repulsive interactions, with registry with high-symmetry binding sites playing a relatively minor role. In contrast, binding at high symmetry sites dominates the ordering at and 
below 0.6 ML and at and above 0.6875 ML. Further investigation of the diversity of structures in the narrow coverage range between these two regimes by DFT or molecular dynamics simulations would provide insight into the balance between adsorbate-substrate and interadsorbate interactions. We concur with Rose et al., that "after decades of extensive research, employing almost every conceivable surface science technique, it is... remarkable that the adsorption of carbon monoxide on metal surfaces continues to yield new information" [9].

\section{Acknowledgement}

This article is based upon work supported in its entirety by the U.S. Department of Energy (DOE), Office of Science, Office of Basic Energy Sciences, Chemical Sciences, Geosciences, \& Biosciences (CSGB) Division. The experiments and analysis by P.X., S.-Y.H., M.G.W. and N.C. were supported by CSGB's Condensed Phase and Interfacial Molecular Science Program. Theoretical calculations by S.L. and P.L were supported by CSGB's Catalysis Science Program, and performed using the computing facility at the Center for Functional Nanomaterials, which is a U.S. DOE Office of Science Facility, at Brookhaven National Laboratory. All of the work was performed under DOE Contract No. DE-SC0012704.

\section{References}

[1] J.C. Tracy, P.W. Palmberg, J. Chem. Phys. 51 (1969) 4852-4862.

[2] H. Conrad, G. Ertl, J. Koch, E.E. Latta, Surf. Sci. 43 (1974) 462-480.

[3] H. Conrad, G. Ertl, J. Küppers, Surf. Sci. 76 (1978) 323-342.

[4] A.M. Bradshaw, F.M. Hoffmann, Surf. Sci. 72 (1978) 513-535. 
[5] H. Ohtani, M.A. Van Hove, G.A. Somorjai, Surf. Sci. 187 (1987) 372-386.

[6] M. Tüshaus, W. Berndt, H. Conrad, A.M. Bradshaw, B. Persson, Appl. Phys. A 51 (1990) 91-98.

[7] T. Gießel, et al., Surf. Sci. 406 (1998) 90-102.

[8] S. Surnev, M. Sock, M.G. Ramsey, F.P. Netzer, M. Wiklund, M. Borg, J.N. Andersen, Surf. Sci. 470 (2000) 171-185.

[9] M.K. Rose, T. Mitsui, J. Dunphy, A. Borg, D.F. Ogletree, M. Salmeron, P. Sautet, Surf. Sci. 512 (2002) 48-60.

[10] C.M. Yim, C.L. Pang, G. Thornton, J. Phys. Chem. C 119 (2015) 22044-22049.

[11] Z. Hooshmand, D. Le, T.S. Rahman, Surf. Sci. 655 (2017) 7-11.

[12] X. Guo, J.T. Yates, Jr., J. Chem. Phys. 90 (1989) 6761-6766.

[13] F.M. Hoffmann, Surf. Sci. Rep. 3 (1983) 107-192.

[14] E. Schweizer, B.N.J. Persson, M. Tüshaus, D. Hoge, A.M. Bradshaw, Surf. Sci. 213 (1989) 49-89.

[15] J.P. Biberian, M.A. Van Hove, Surf. Sci. 138 (1984) 361-389.

[16] A. Bondi, J. Phys. Chem. 68 (1964) 441-451.

[17] D. Loffreda, D. Simon, P. Sautet, Surf. Sci. 425 (1999) 68-80.

[18] C.M. Yim, C.L. Pang, D.S. Humphrey, C.A. Muryn, K. Schulte, R. Perez, G. Thornton, Faraday Discuss. 162 (2013) 191-200.

[19] K. Honkala, P. Pirilä, K. Laasonen, Surf. Sci. 489 (2001) 72-82.

[20] N.M. Martin, M. Van den Bossche, H. Grönbeck, C. Hakanoglu, F. Zhang, T. Li, J. Gustafson, J.F. Weaver, E. Lundgren, J. Phys. Chem. C 118 (2014) 1118-1128.

[21] C.M. Yim, C.L. Pang, D.R. Hermoso, C.M. Dover, C.A. Muryn, F. Maccherozzi, S.S. Dhesi, R. Pérez, G. Thornton, Proc. Natl. Acad. Sci. 112 (2015) 7903-7908.

[22] W.K. Kuhn, J. Szanyi, D.W. Goodman, Surf. Sci. 274 (1992) L611-L618. 
[23] H. Unterhalt, G. Rupprechter, H.-J. Freund, J. Phys. Chem. B 106 (2002) 356-367.

[24] M. Morkel, G. Rupprechter, H.-J. Freund, J. Chem. Phys. 119 (2003) 10853-10866.

[25] P. Szymanski, A.L. Harris, N. Camillone, III, J. Chem. Phys. 126 (2007) 214709.

[26] P. Szymanski, A.L. Harris, N. Camillone, III, Surf. Sci. 601 (2007) 3335-3349.

[27] G. Kresse, J. Furthmüller, Phys. Rev. B 54 (1996) 11169-11186.

[28] G. Kresse, J. Hafner, Phys. Rev. B 47 (1993) 558-561.

[29] G. Kresse, J. Hafner, Phys. Rev. B 48 (1993) 13115-13118.

[30] G. Kresse, J. Hafner, Phys. Rev. B 49 (1994) 14251-14269.

[31] G. Kresse, J. Furthmüller, Comp. Mater. Sci. 6 (1996) 15-50.

[32] P.E. Blöchl, Phys. Rev. B 50 (1994) 17953-17979.

[33] J.P. Perdew, Y. Wang, Phys. Rev. B 45 (1992) 13244-13249.

[34] J.P. Perdew, K. Burke, M. Ernzerhof, Phys. Rev. Lett. 77 (1996) 3865-3868.

[35] A. Tkatchenko, M. Scheffler, Phys. Rev. Lett. 102 (2009) 073005.

[36] H.J. Monkhorst, J.D. Pack, Phys. Rev. B 13 (1976) 5188-5192.

[37] M. Morkel, H. Unterhalt, M. Salmeron, G. Rupprechter, H.-J. Freund, Surf. Sci. 532-535 (2003) 103-107.

[38] G. Rupprechter, Adv. Catal. 51 (2007) 133-263.

[39] M. Gajdoš, A. Eichler, J. Hafner, J. Phys. Condens. Matter 16 (2004) 1141-1164. 

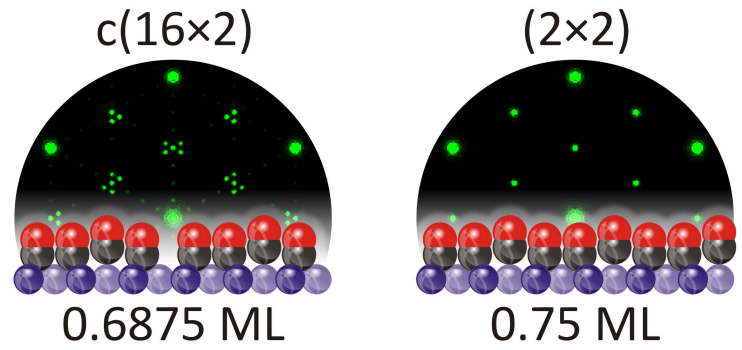